Published in final edited form as:

Sci Transl Med. 2011 August 31; 3(98): 98fs2. doi:10.1126/scitranslmed.3002806.

\title{
Imaging Cardiac Arrhythmias
}

\author{
Kalyanam Shivkumar ${ }^{1,{ }^{*}}$ and Sanjiv M. Narayan ${ }^{1,2,3}$ \\ 1 University of California, Los Angeles, Cardiac Arrhythmia Center, UCLA Health System, David \\ Ge en School of Medicine at UCLA, Los Angeles, CA 90095, USA. \\ 2University of California, San Diego School of Medicine, San Diego, CA 92103, USA. \\ ${ }^{3}$ San Diego VA Health System, La Jolla, CA 92161, USA.
}

\begin{abstract}
An imaging modality that combines electrocardiography and computed tomography identifies the origins of ventricular tachycardias (Wang et al., this issue).
\end{abstract}

\section{ADVANCING THE ELECTROCARDIOGRAM}

In the rapidly changing world of clinical medicine, few technologies become rmly established in practice and then continue as the basis for continued innovation for over a century. The electrocardiogram (ECG), which measures microvolt electrical potentials at the surface of the human body to infer electrical activity of the heart, is one such technology. At the turn of the past century, Willem Einthoven developed the string galvanometer to amplify and display minute potentials from the heart, ushering in the era of electrocardiography. The technique quickly established itself as a tool to study heart physiology and as an indispensable and practical diagnostic method for diseases of the heart as well as cardiac manifestations of lung, endocrine, and metabolic diseases (1).

The ECG provides an instantaneous and reproducible vectorial display of electrical activity throughout the heart, including the atria and the ventricles [both the activation and subsequent recovery (repolarization)]. In its current form, the ECG provides an easily accessible, universally available tool for the recognition of slow and fast, regular and irregular heart rhythms (arrhythmias) and corresponding relationships between activation of the atria and ventricles. Accordingly, it has become central to monitoring health and disease in diverse patient care settings, from outpatient clinics to ambulances. Nevertheless, modern medicine continues to demand that the ECG improve its spatial resolution—beyond identifying from which of the two atria or ventricles the electrical activation arises. Further, clinicians would like to know where precisely electrical activation originates for any given arrhythmia. The need to map the precise origins of abnormal, disease-causing electrical activity in the heart has been spurred by exponential growth in the field of invasive electrophysiology, in which probes are advanced to the heart from the peripheral vasculature to diagnose the nature and origin of arrhythmias. Contemporary interventional electrophysiology uses electrical stimulation (also known as "pacing") to induce arrhythmias and then perform carefully targeted ablation (delivery of radiofrequency energy) to treat arrhythmias at their sites of origin.

*Corresponding author. kshivkumar@mednet.ucla.edu.

Competing interests: K.S. is an inventor of intellectual property for invasive cardiac procedures, and S.M.N. is an inventor of intellectual property for invasive mapping, both of which are owned by the University of California Regents. 
In this issue of Science Translational Medicine, Wang et al. (2) extend the use of ECG in the clinic, using a technique called electrocardiographic imaging (ECGI). The authors apply ECGI to noninvasively map activation paths on the heart in order to identify the exact site of origin of potentially life-threatening ventricular tachycardias (VTs) (Fig. 1).

\section{ECG MEETS IMAGING TECHNOLOGY}

The standard method for mapping ventricular arrhythmias involves placing catheters that record local electrical activity in the heart, followed by validation of that information against the ECG and construction of maps of electrical propagation through the heart. This process requires data sampling from the heart during an invasive procedure. The great strength of ECGI is its ability to obtain a "map" of electrical activation at the bedside before the therapeutic catheter ablation procedure, thus allowing the physician to subsequently focus on areas of interest.

Wang and colleagues selected a series of patients with frequently encountered arrhythmias so as to validate ECGI through correlation with invasive mapping and successful ablation (2). The study population of 25 patients represented a range of VTs, or abnormal rapid rhythms originating in the ventricles. For ECGI, each patient underwent detailed body surface recordings from 256 electrodes during VT, which were registered in the coordinate space of a previously acquired computed tomogram (CT) to provide anatomical information for computational analysis (Fig. 1). This method allowed the generation of "maps" of electrical activation on the heart, from which the origin of the VT could be identified. Each patient also underwent a traditional, invasive electrophysiological (EP) study, in which multiple probes were advanced to the heart from peripheral vasculature, to map the origin of VT. The arrhythmia was then targeted for curative ablation.

The authors were able to validate the precision of ECGI for locating the site of origin of VT to the correct ventricle (left or right) in each patient and to the precise site of origin in more than $90 \%$ of the patients, as confirmed with the invasive EP study (2). Even in discrepant cases, the sites of origin identified by ECGI lay adjacent to sites identified from traditional invasive mapping. Additionally, ECGI-derived maps recapitulated features of electrical signals also found on invasive mapping, including regions of ventricular scar, double potentials, and fractionated signals. Thus, the ECGI approach to noninvasively map VTs described by Wang and colleagues holds great promise for improved identification and treatment of life-threatening cardiac arrhythmias.

\section{REVERSING THE INVERSE PROBLEM}

In developing ECGI and bringing it to clinical fruition, the work of Rudy and colleagues has solved a vexing issue in cardiology: the inverse problem of mapping the presence or absence of deflections on the ECG to potentials on the surface of the heart, or epicardium (3).

Mathematically, this is a degenerate problem with multiple solutions because each ECG lead records an average of potentials from all heart regions, weighted spatially by proximity to the lead, and also factors in the electrical conductance of heterogeneous tissue, which is balanced by activation paths away from the lead (4). In contrast, vectorial analysis of the traditional ECG remains a technology of approximation.

The ECGI solution to the inverse problem is steeped in fundamental studies of the mechanisms underlying activation and recovery at cellular (5) and subcellular levels and their subsequent translation into numerical computational models (6). Conduction paths are then modeled by registering voxels from a patient-specific $\mathrm{CT}$ of the chest, which visualizes the torso surface, lungs, and other mediastinal structures, in the axis-system of the surface ECG leads (7). ECGI also uses higher-density spatial sampling of the chest, increased from 
the 12 traditional ECG leads to a "body surface map" of 256 electrodes acquired concurrently with the chest CT during the arrhythmia. Computational methods then use these data to solve for electrical activation on the epicardium and triangulate the site of origin of a rhythm disturbance, such as VT, from the body-surface signals. is approach can be performed rapidly (in tens of minutes), as exemplified during the clinical procedure in several patients reported by Wang et al. (2).

The early saga of electrocardiography was an expedition led by Einthoven and visionary collaborators who bridged the basic science laboratory and clinical wards to forge the indispensable tool that is the contemporary ECG. Such collaboration was often difficult; indeed, at one point a telephonic cable link between Einthoven's lab in Leiden and the nearby hospital was severed owing to an inability of clinicians and scientists to work as a team (8). Nevertheless, their persistence in performing bench-to-bedside translation, well before the term "translation" was even coined, resulted in the groundbreaking modality of ECG. Recent decades have seen remarkable progress in understanding intricate cellular and molecular processes in biology, whose true merit will be realized only through similar translation to clinical practice. Wang et al. elegantly show translation of fundamental ECG studies to a real-world clinical application of ECGI (2), which has the potential to transform the management and ablation of human ventricular tachycardias. The ability to obtain a high-quality electrical "roadmap" with ECGI is likely to make future journeys into the heart for therapeutics a more predictable experience.

\section{REFERENCES AND NOTES}

1. Fisch C. Centennial of the string galvanometer and the electrocardiogram. J. Am. Coll. Cardiol. 2000; 36:1737-1745. [PubMed: 11092639]

2. Wang Y, Cuculich PS, Zhang J, Desouza KA, Vijaya-kumar R, Chen J, Faddis MN, Lindsay BD, Smith TW, Rudy Y. Noninvasive electroanatomic mapping of human ventricular arrhythmias with electrocardiographic imaging (ECGI). Sci. Transl. Med. 2011; 3:98ra84.

3. Ramanathan C, Ghanem RN, Jia P, Ryu K, Rudy Y. Noninvasive electrocardiographic imaging for cardiac electrophysiology and arrhythmia. Nat. Med. 2004; 10:422-428. [PubMed: 15034569]

4. Nash MP, Pullan AJ. Challenges facing validation of noninvasive electrical imaging of the heart. Ann. Noninvasive Electrocardiol. 2005; 10:73-82. [PubMed: 15649241]

5. Gaur N, Rudy Y. Multiscale modeling of calcium cycling in cardiac ventricular myocyte: macroscopic consequences of microscopic dyadic function. Biophys. J. 2011; 100:2904-2912. [PubMed: 21689523]

6. O’Hara T, Virág L, Varró A, Rudy Y. Simulation of the undiseased human cardiac ventricular action potential: Model formulation and experimental validation. PLoS Comput. Biol. 2011; 7:e1002061. [PubMed: 21637795]

7. Ramanathan C, Rudy Y. Electrocardiographic imaging: I. Effect of torso inhomogeneities on body surface electrocardiographic potentials. J. Cardiovasc. Electrophysiol. 2001; 12:229-240. [PubMed: 11232624]

8. Wellens HJ. Bishop lecture. The electrocardiogram 80 years after Einthoven. J. Am. Coll. Cardiol. 1986; 7:484-491. [PubMed: 3512659] 


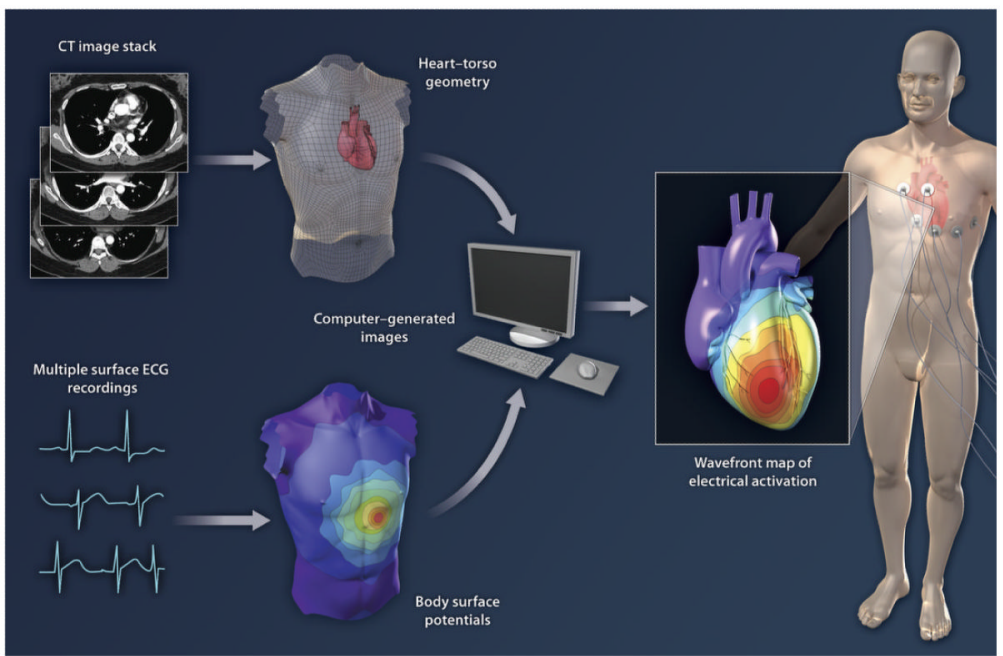

Fig. 1.

ECG combines with CT imaging to produce 3D maps of electrical activity on the surface of the human heart. Multiple surface electrodes are used to generate ECG data for body surface potential mapping. High-resolution CT images provide information on the anatomy and orientation of the heart and chest wall. Electrical and imaging data are processed by the ECGI algorithm to provide a map of electrical activation. 